\title{
A Cross-Sectional Study Comparing the Frequency of Drug Interactions After Adding Simeprevir- or Sofosbuvir-Containing Therapy to Medication Profiles of Hepatitis C Monoinfected Patients
}

\author{
Nimish Patel $\cdot$ Mona Nasiri $\cdot$ Arden Koroglu $\cdot$ Steven Bliss • \\ Melissa Davis · Louise-Anne McNutt • Christopher Miller
}

To view enhanced content go to www.infectiousdiseases-open.com Received: November 10, 2014 / Published online: January 28, 2015

(C) The Author(s) 2015. This article is published with open access at Springerlink.com

\section{ABSTRACT}

Introduction: This study compares the expected occurrence of contraindicated drugdrug interactions (XDDIs) when simeprevir (SIM)- or sofosbuvir (SOF)-containing therapy is added to medication profiles of patients with hepatitis $\mathrm{C}(\mathrm{HCV})$ monoinfection to quantify, in relative terms, the population-based risk of XDDIs. Second, this study identified the predictors of XDDIs when HCV therapies are added to medication profiles.

Methods: A cross-sectional study was performed among Veterans' Affairs patients.

Electronic supplementary material The online version of this article (doi:10.1007/s40121-015-0058-x) contains supplementary material, which is available to authorized users.

N. Patel $(\bowtie) \cdot$ M. Nasiri · A. Koroglu · S. Bliss ·

M. Davis

Albany College of Pharmacy and Health Sciences, 106 New Scotland Avenue, Albany, NY 12208, USA e-mail: nimish.patel@acphs.edu

L.-A. McNutt

Institute for Health and the Environment,

University at Albany, State University of New York,

Albany, NY, USA

C. Miller

Departments of Pharmacy and Medicine, Upstate

University Hospital, Syracuse, NY, USA
Inclusion criteria were: (1) age $\geq 18$ years, (2) HCV infection, and (3) availability of a medication list. Patients with human immunodeficiency virus were excluded. Demographics, comorbidities, year of $\mathrm{HCV}$ diagnosis, and most recent medication list were collected from medical records. The primary outcome was the presence of XDDIs involving $\mathrm{HCV}$ therapy and the medications in the patient's home medication list after the addition of either SIM- or SOF-containing regimens. To define XDDIs, Lexi-Interact drug interaction software was used.

Results: 4,251 patients were included. The prevalence of XDDIs involving SIM- or SOFcontaining therapy were $12.6 \%$ and $4.7 \%$ $(p<0.001)$, respectively. In multivariable analyses examining the predictors of XDDIs involving SIM-containing therapy, the only medication-related predictor was use of $\geq 6$ home medications (odds ratio OR 4.58, 95\% confidence interval CI 3.54-5.20, $p<0.001$ ). Similarly, use of $\geq 6$ home medications was also the only variable associated with an increased probability of XDDI involving SOFcontaining therapy (OR 3.83, 95\% CI 2.57-5.70, $p<0.001)$. 
Conclusions: Sofosbuvir-containing therapy had a lower frequency of XDDIs than SIMcontaining therapy. Polypharmacy with various classes of home medications predicted XDDIs involving SIM- or SOF-containing therapy.

Keywords: Drug interactions; Epidemiology; Hepatitis C; Pharmacy

\section{INTRODUCTION}

Several new medications have become available to treat chronic hepatitis $\mathrm{C}(\mathrm{HCV})$ infection. These include drugs from several new directacting antiviral medication classes that are inhibitors of non-structural (NS)3/4A serine protease and NS5B polymerase $[1,2]$. The treatment of chronic $\mathrm{HCV}$ infection has become more widely available given the approval of these medications. The clinical guidance for treating chronic $\mathrm{HCV}$ infection now typically includes multiple drugs: sofosbuvir (SOF) or simeprevir (SIM) in combination with other medications [3]. The simultaneous use of multiple medications, polypharmacy, is associated with an increased likelihood of contraindicated drug-drug interactions (XDDI) [4].

Important safety concerns arise when dealing with polypharmacy due to the increased potential of XDDIs among the chronic HCV-infected population. The majority of patients with chronic hepatitis $\mathrm{C}$ are between the ages of 50-70 years and, as such, typically have developed age-related comorbidities that often necessitate management with chronic maintenance medications $[5,6]$. Some of these medications may interact with HCV therapy and could potentially result in poor treatment outcomes including toxicity and incomplete therapy, which may lead to a diminished probability of achieving a sustained virologic response, or the development of resistance $[7,8]$. However, there is little data quantifying the excess frequency of XDDIs that occur between SIM- or SOFcontaining treatment regimens when added to the existing medication profiles of $\mathrm{HCV}$ monoinfected patients. The majority of this work has been focused on patients with human immunodeficiency virus (HIV)/HCV coinfection $[9,10]$. An understanding of the frequency and predictors of XDDIs when either SIM- or SOF-containing therapy is considered for initiation will aid clinicians in managing the most appropriate therapy for a given patient with HCV monoinfection.

This study compares the expected occurrence of XDDIs when SIM- or SOFcontaining therapy is added to medication profiles of patients with HCV monoinfection. Secondly, this study identifies the predictors of XDDIs when SIM- and SOF-containing therapies are added to patient's home medication lists.

\section{METHODS}

A cross-sectional study was performed among HCV monoinfected Veterans' Affairs patients receiving care between 2000 and 2013 in the Upstate New York Veterans' Healthcare Administration (VHA) or Veterans' Integrated Service Network Region 2 (VISN-2). Patients were identified using the following International Classification of Diseases 9th Revision (ICD-9) codes related to hepatitis C infection: 070.41 (acute hepatitis $\mathrm{C}$ with hepatic coma), 070.44 (chronic hepatitis C with hepatic coma), 070.51 (acute hepatitis C without mention of hepatic coma), 070.54 (chronic hepatitis $\mathrm{C}$ without mention of hepatic coma), 070.70 (unspecified hepatitis C 
without hepatic coma), and 070.71 (unspecified hepatitis $\mathrm{C}$ with hepatic coma). Each of these patients was screened to confirm HCV infection and for the following inclusion criteria: (1) age $\geq 18$ years, (2) laboratory-confirmed HCV infection, and (3) availability of a medication list. Patients with (HIV) were excluded because of the strong influence of antiretroviral therapy on the probability of XDDIs [11].

Data were obtained from the patients' medical records related to demographics, concomitant medical problems, year HCV infection was diagnosed, and medication list. Demographic characteristics included age, sex, race (African American, Hispanic, Caucasian, and other), height and weight. Concomitant medical problems were obtained from the clinical progress note. The most recent medication list in the patients' medical records was obtained and used in this analysis. For each medication, the drug name, dose, and frequency were recorded. Polypharmacy was defined as the use of multiple medications.

The outcome of interest was the presence of XDDIs involving HCV therapy and the medications in the patient's most recent medication list (home medications) after the addition of either SIM- or SOF-containing regimens. SIM, pegylated interferon, and ribavirin were used to define SIM-containing therapy. Analogously, SOF-containing therapy was defined as SOF, pegylated interferon and ribavirin.

To define XDDIs, Lexi-Interact drug interaction software (Lexicomp, Hudson, USA) was used [12]. The drug-drug interactions that Lexi-Interact ranked as X-rated (contraindicated) were considered XDDIs. All of the patients' home medications were entered into Lexi-Interact, and the number of XDDIs was recorded. Then, SIM-containing therapy was added to the existing home medications and the number of identified additional XDDIs was recorded. Finally, SIM-containing therapy was removed from Lexi-Interact and replaced with SOF-containing therapy, and the number of additional XDDIs was recorded.

To assess the data for the first study objective, McNemar's test was used to compare the frequencies of XDDIs after adding SIM- and SOF-containing therapy to patients' home medication profiles. For study objective \#2 (predictors of XDDI involving SIM- and SOFcontaining therapy), bivariate analyses were performed to assess the relationship between the clinical covariates and XDDIs after the addition of a SIM- and SOF-containing HCV regimen to the patients' medication lists. The $\chi^{2}$ or Fisher's exact tests were used to evaluate categorical variables. The Student's $T$ or MannWhitney $U$ tests were used to compare continuous data. Variables present in more than $5 \%$ of the population and associated with an XDDI involving SIM- and SOF-containing therapy in the bivariate analyses $(p<0.25)$ were entered into the multivariate regression analyses. A backward stepwise approach was used to identify the most parsimonious models. Effect modification was evaluated by including interaction terms in the multivariate models. Classification and regression tree (CART) analysis was used to identify thresholds in continuous variables at which the likelihood of an XDDI distinctly differs. All calculations were computed using SPSS version 11.5 (SPSS Inc, Chicago, IL, USA), SAS version 9.3 (SAS Institute, Cary, NC, USA), and CART software (Salford Systems, San Diego, CA, USA).

This article does not contain any new studies with human or animal subjects performed by any of the authors. 


\section{RESULTS}

There were 4,251 patients who met inclusion criteria. The mean \pm standard deviation (SD) age was $59.8 \pm 7.6$ years. The majority $(n=4,102,96.5 \%)$ of patients were male. Caucasian race $(n=2,476,58.2 \%)$ was observed most frequently, followed by African American $(n=1,219,28.7 \%)$, other $(n=438$, $10.3 \%)$, and Hispanic $(n=118,2.8 \%)$. The median (interquartile range, IQR) number of years since HCV diagnosis was 12 (8-14). The median (IQR) numbers of comorbidities and home medications used were 7 (5-10) and 7 (4-11), respectively. There were 477 (11.2\%) patients with cirrhosis.

The prevalence of XDDI before the addition of SIM- or SOF-containing therapy was $16.7 \%$ $(n=709)$. After the addition of SIM- and SOFcontaining therapy, the overall prevalence of XDDIs significantly increased to $25.9 \%$ $(n=1,103)$ and $19.9 \% \quad(n=844, p<0.001)$, respectively. When restricting interactions to only those involving SIM- or SOF-containing therapy (Fig. 1), the prevalence of XDDIs after the addition of these drugs was $12.6 \%(n=535)$ and $4.7 \%(n=201, p<0.001)$ for SIM- and SOFcontaining therapies, respectively. Upon stratification by the CART-derived breakpoints ( $<$ or $\geq 6$ home medications and having $<$ or $\geq 10$ comorbidities), there were significant differences in the frequency of XDDIs involving $\mathrm{HCV}$ therapy between SIM- and SOF-containing regimens. The differences within each of the strata were consistent with the overall comparison in that SIM-containing drugs tended to be associated with a greater likelihood of an XDDI.

The bivariate analyses of XDDIs involving HCV medications after the addition of SIM- and SOF-containing therapies are described in Table 1. The variables associated with XDDIs involving SIM-containing therapy were age, weight, time since HCV diagnosis, total number of home medications, and total number of comorbidities. Specific home medication classes associated with XDDI involving SIM-containing therapy were antipsychotics, antibiotics, antiepileptics, azole antifungals, statins, calcium channel blockers, corticosteroids, central nervous system (CNS) depressants, erectile dysfunction drugs, methadone, antiarrhythmic, corticosteroids, alpha or muscarinic-3 blockers, and beta-2 agonists. Specific comorbidities associated with XDDI involving SIM-containing therapy were seizure disorder, arrhythmias, heart failure, heart disease, chronic kidney disease, recreational drug use, alcoholism, cancer, dementia, chronic obstructive pulmonary disorder, neuropathy, hypertension, organ transplant, and anemia.

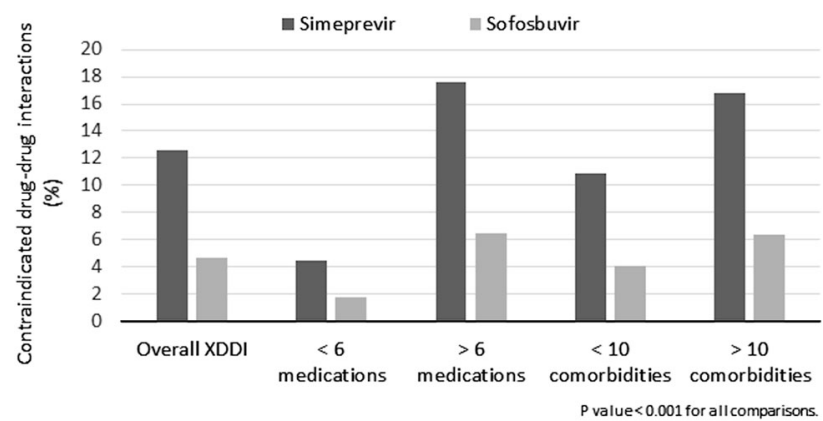

Fig. 1 Prevalence of contraindicated drug-drug interactions (XDDI) involving hepatitis C therapy after addition of simeprevir- and sofosbuvir-containing therapy 


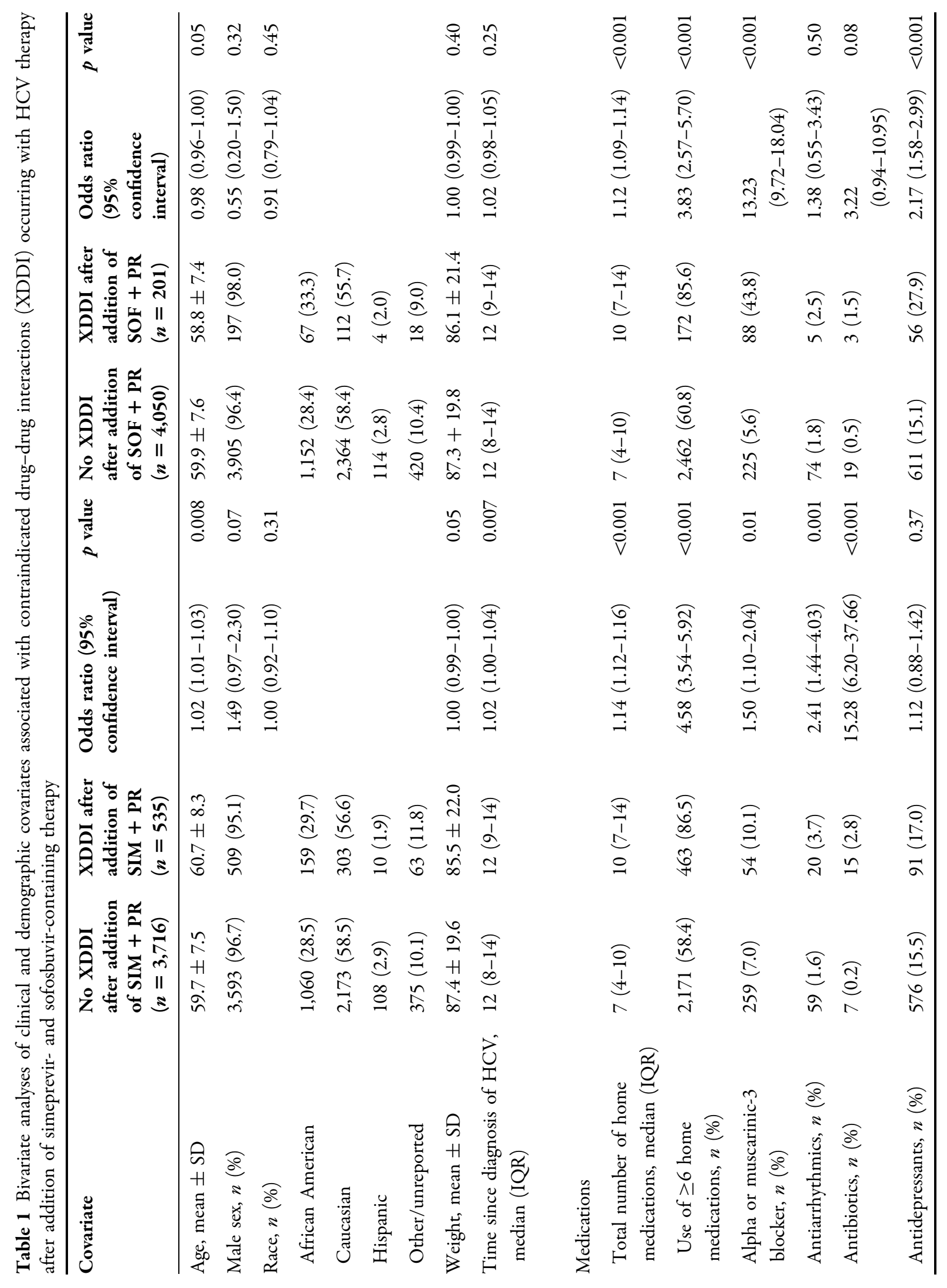




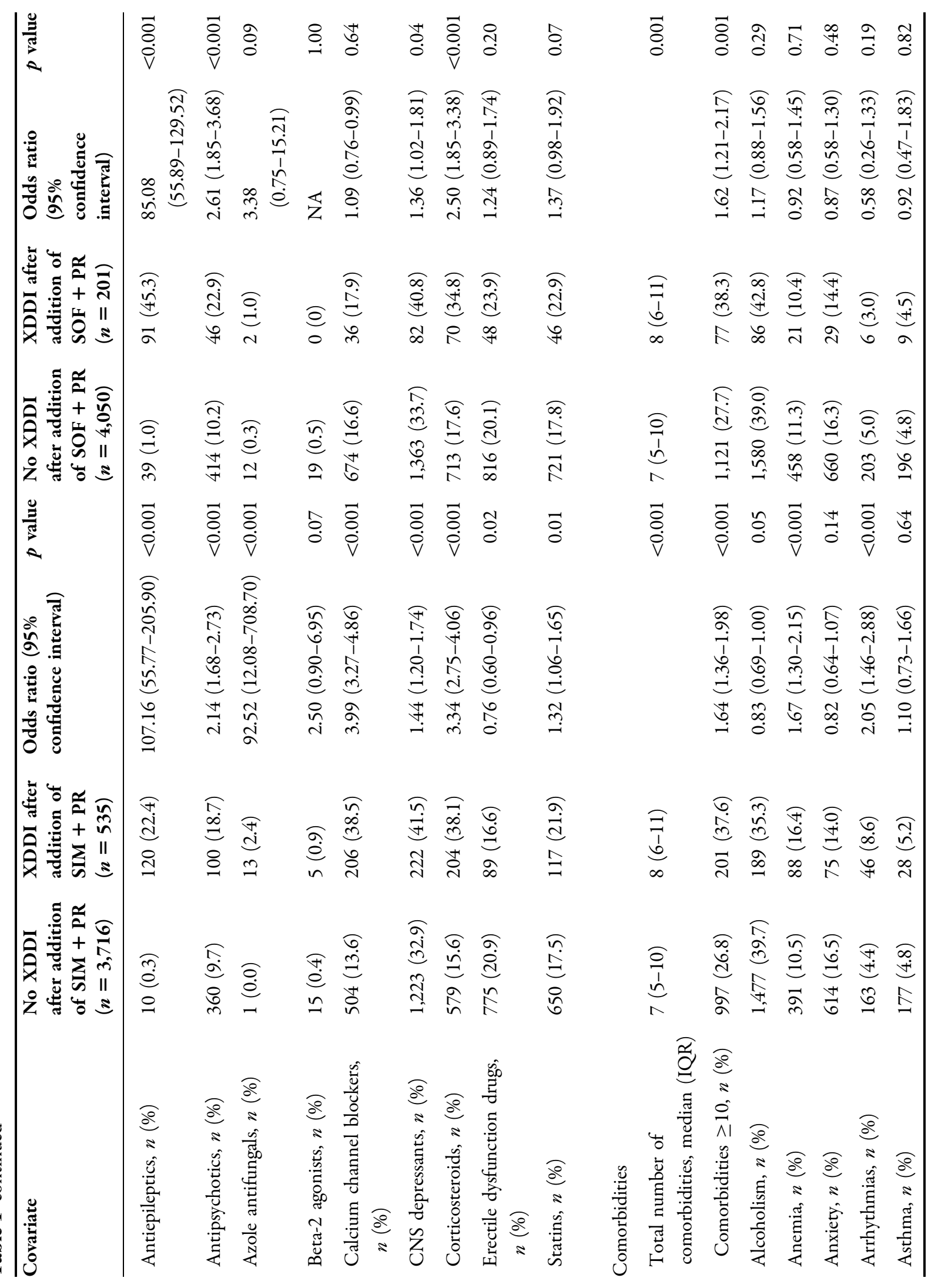




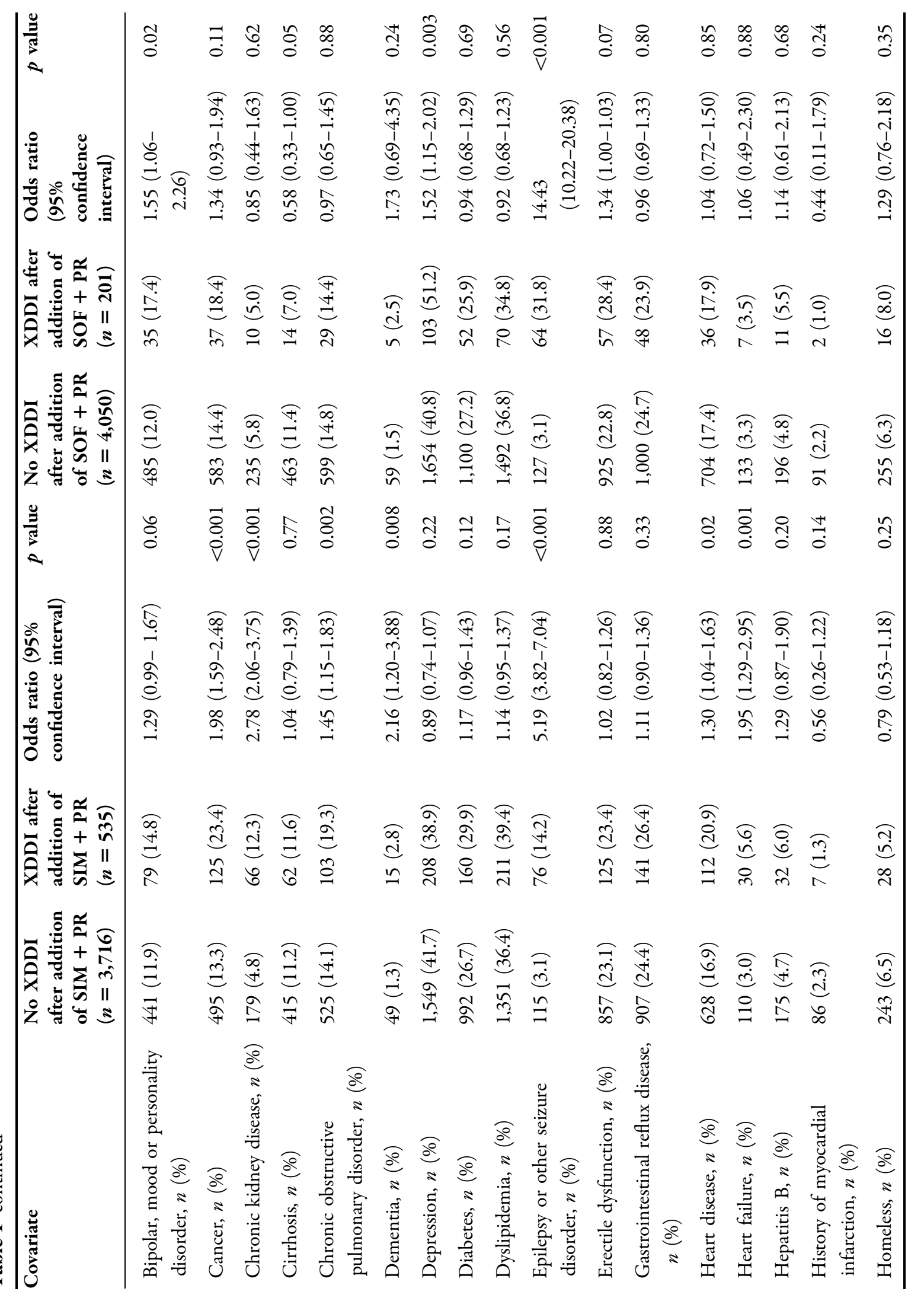




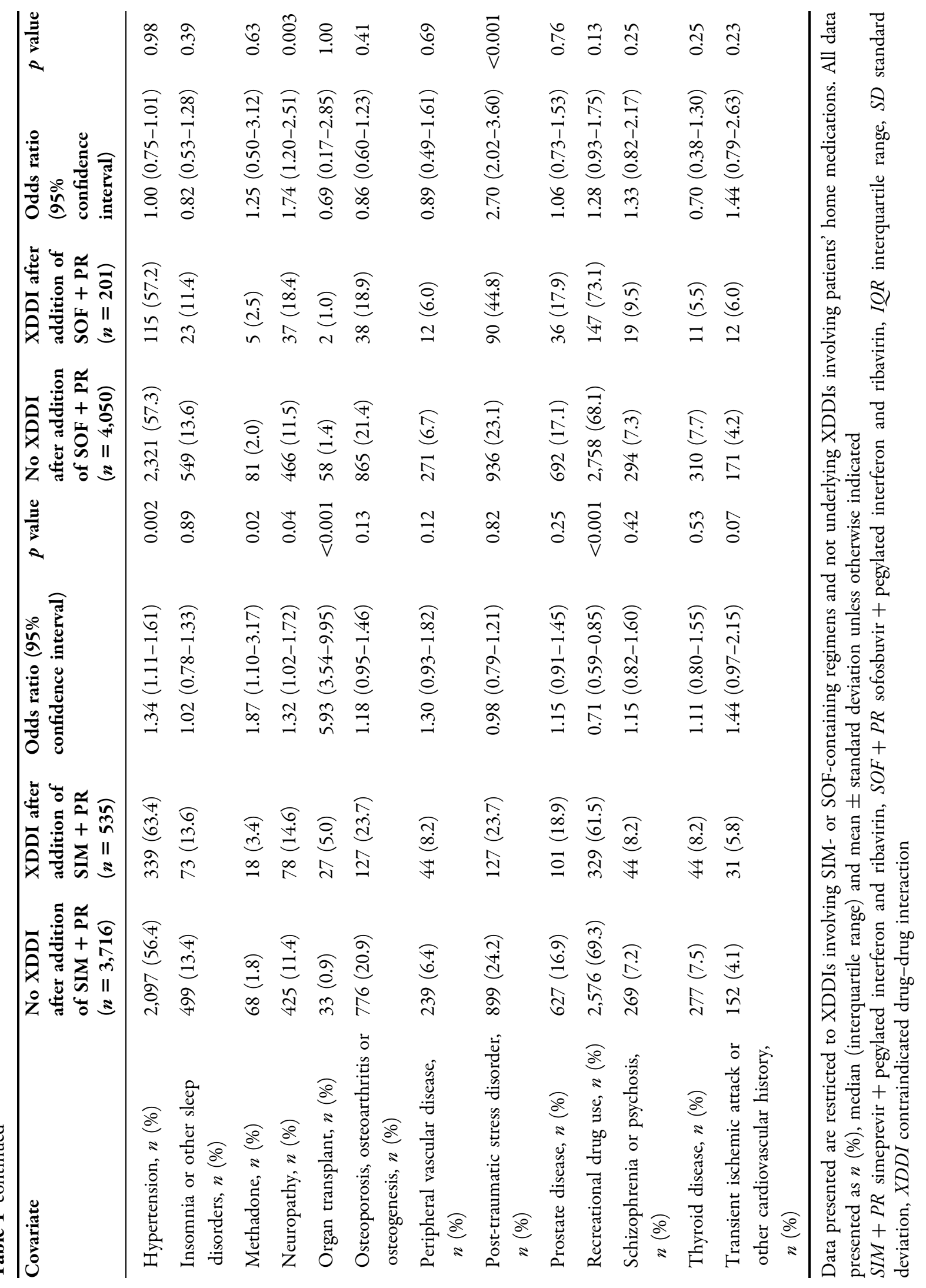


Fewer variables were significantly associated with XDDI involving SOF-containing therapy. These included age, total number of home medications, antipsychotics, antidepressants, antiepileptic drugs, CNS depressants, corticosteroids, alpha or muscarinic-3 blocker, total number of comorbidities, bipolar/mood/ personality disorder, depression, seizure disorder, post-traumatic stress disorder, neuropathy, and cirrhosis. The use of $\geq 6$ home medications and having $\geq 10$ comorbidities was associated with an increased frequency of XDDI involving either SIM- or SOF-containing therapy.

In multivariable analyses examining the predictors of XDDIs involving SIM-containing therapy, the only medication-related predictor was use of $\geq 6$ home medications (odds ratio OR 4.58 , 95\% confidence interval CI 3.54-5.20, $p<0.001)$. Similarly, the use of $\geq 6$ home medications was also the only variable associated with an increased probability of XDDI involving SOF-containing therapy (OR $3.83,95 \%$ CI 2.57-5.70, $p<0.001)$. To make the models more specific, the use of $\geq 6$ home medications was replaced with the medication classes associated with XDDI involving either SIM- or SOF-containing therapy. The results of these refined multivariable models are displayed in Table 2. Variables independently associated with XDDI involving SIM-containing therapy were calcium channel blockers, corticosteroids, antipsychotics, antiarrhythmic, and CNS depressants. Variables independently associated with XDDIs involving SOFcontaining therapy were corticosteroids, antipsychotics, antidepressants, and statins.

\section{DISCUSSION}

Therapies containing SIM or SOF are associated with a high cost and it is imperative that clinicians take appropriate measures to ensure
Table 2 Variables independently associated with contraindicated drug-drug interactions after the addition of simeprevir- or sofosbuvir-containing hepatitis $\mathrm{C}$ therapy

\begin{tabular}{llll}
\hline Variable & $\begin{array}{l}\text { Odds } \\
\text { ratio }\end{array}$ & $\begin{array}{l}95 \% \\
\text { confidence } \\
\text { interval }\end{array}$ & $p$ value \\
\hline
\end{tabular}

XDDI after addition of simeprevir-containing therapy

\begin{tabular}{lccc}
$\begin{array}{l}\text { Calcium channel } \\
\text { blockers }\end{array}$ & 4.15 & $3.38-5.11$ & $<0.001$ \\
Corticosteroids & 3.41 & $2.78-4.19$ & $<0.001$ \\
Antipsychotics & 2.53 & $1.96-3.28$ & $<0.001$ \\
Antiarrhythmics & 2.23 & $1.28-3.89$ & 0.005 \\
Central nervous & 1.32 & $1.09-1.61$ & 0.005 \\
system depressants & & & \\
XDDI after addition of sofosbuvir-containing therapy \\
Corticosteroids & 2.54 & $1.87-3.44$ & 0.001 \\
Antipsychotics & 2.44 & $1.71-3.48$ & 0.001 \\
Antidepressants & 1.90 & $1.37-2.64$ & 0.001 \\
Statins & 1.40 & $1.00-1.97$ & 0.05 \\
\hline
\end{tabular}

that patients have the highest likelihood of completing therapy, achieving a sustained virologic response and minimizing adverse events [13]. In some situations, adverse events that are a function of XDDIs can be prevented by proactively identifying patients at risk for XDDIs prior to prescribing SIM or SOFcontaining therapy and choosing the type of therapy that is associated with a lower probability or using them in patients who do not have risk factors for XDDIs [14]. This intervention, coupled with optimal drug adherence, can contribute to the probability of a good treatment outcome.

The majority of other population-based drug-drug interaction studies have been focused on patients with HIV/HCV coinfection $[9,10]$. The present study, one of the largest population-based drug-drug interaction studies, focused on patients with HCV monoinfection. 
The results presented here are consistent with other publications $[4,9,10,15]$. It was observed that polypharmacy was an issue among patients with $\mathrm{HCV}$ monoinfection and these patients are vulnerable to the effects of XDDI. Patients in the study were using a median of seven home medications, and over $15 \%$ of patients had XDDIs involving medications that they were already taking and before the addition of SIMor SOF-containing therapy. This is important because several other patient populations (HIV, transplant, dialysis, etc.) are considered high risk for XDDIs and now, given these data, patients with HCV monoinfection should be considered as well [15-18].

Patients with HCV monoinfection may need pharmacotherapeutic intervention to resolve any existing XDDIs before being prescribed SIM- or SOF-containing therapy. It was also observed that SIM-containing regimens were associated with nearly triple the likelihood of XDDIs compared to SOF-containing regimens. These results are plausible given that SIM is a CYP3A hepatic substrate and intestinal inhibitor [1]. There may be altered exposure of both SIM and other drugs that also utilize these enzymatic pathways when used concomitantly [1]. Conversely, SOF is metabolized by P-glycoprotein (P-gp), a less ubiquitously utilized metabolic pathway, and SOF exposure may be altered in the presence of other P-gp inducers/inhibitors [2]. Many XDDIs involving either type of HCV therapy were predicted by use of various drug classes. These data demonstrate the need for a pharmacist to review patients' medications before $\mathrm{HCV}$ therapy is prescribed.

To appropriately interpret these data, the following considerations should be kept in mind. First, exposure to SIM- and SOFcontaining HCV therapy was completely theoretical. No patients in the study actually received SIM- or SOF-containing therapy. Rather, SIM- and SOF-containing therapy was added to the home medication lists of the patients in the study to assess what proportion would have an XDDI if either of these regimens were prescribed. This is strength of the paper, because it demonstrates how a counterfactual study design allows the evaluation of an outcome of interest in the same patient under two exposure conditions. Second, patients with HIV were excluded because antiretroviral therapy is an exceptionally strong predictor of drug-drug interactions [11]. Apart from the use of anti-retrovirals, it was also unclear if medication use patterns differed between coinfected and monoinfected patients. Because of these issues, it was felt that inclusion of patients with HIV/HCV coinfection would mask some of the non-HIV-related predictors of XDDI involving SIM- or SOF-containing therapy. To preserve internal validity, the choice to restrict the study population to only those with $\mathrm{HCV}$ monoinfection was made. Third, liver performance [fibrosis/alanine transaminase (ALT), etc.] was not known and this, as well as other markers or disease severity, may be associated with higher medication use and subsequent risk of XDDIs. Future studies should attempt to assess the contribution of markers of disease severity and determine if variables such as time since HCV diagnosis are truly associated with a higher probability of medication use and XDDIs. Fourth, some of the predictors, particularly for XDDI involving SOFcontaining therapy, are not drug classes that are contraindicated with SOF-containing therapy in the product labeling [2]. It is important to note that patients receiving these drug classes were more likely to have an XDDI involving SOFcontaining therapy, although these classes are contraindicated themselves. More than likely, these predictors may be indicative of XDDIs 
that may exist prior to the addition of $\mathrm{HCV}$ therapy or a proxy for other variables such as disease severity, whereby patients may require the use of several medications to control other underlying comorbidities. Finally, the hepatitis $\mathrm{C}$ armamentarium is changing rapidly. The regimens we evaluated contained interferon and ribavirin in combination with either SIM or SOF. Future HCV treatment regimens may not contain some or all of these components. It is important to note that interferon and ribavirin are associated with few XDDIs and the majority of interactions were driven in large part due to SIM or SOF. This study will need to be repeated in the future with emerging agents like ledipasvir, ombitasvir, dasabuvir, and daclatasvir.

\section{CONCLUSION}

In summary, the results of this study found that HCV monoinfected patients are at high risk for XDDIs, and SOF-containing therapy had a lower frequency of XDDIs than SIM-containing therapy. Polypharmacy with various classes of home medications predicted XDDIs involving SIM- or SOF-containing therapy. Clinicians prescribing either of these treatment regimens should thoroughly evaluate patients' existing medications to avoid potential XDDIs.

\section{ACKNOWLEDGMENTS}

This material is based upon work partially supported by the Office of Research and Development, Department of Veterans Affairs. This article has greatly benefited from the thoughtful editing of Allison Krug and the data entry of Michael Veve.

No funding or sponsorship was received for this or publication of this article.
All named authors meet the ICMJE criteria for authorship for this manuscript, take responsibility for the integrity of the work as a whole, and have given final approval for the version to be published.

Conflict of interest. Mona Nasiri, Arden Koroglu, Steven Bliss, Melissa Davis, LouiseAnne McNutt, and Christopher Miller declare that they have no conflicts of interest.

Dr. Patel has received research grant support for other studies from Gilead Sciences. Dr. Patel owns five shares of stock in Gilead Sciences.

Compliance with ethics. This article does not contain any new studies with human or animal subjects performed by any of the authors.

Open Access. This article is distributed under the terms of the Creative Commons Attribution Noncommercial License which permits any noncommercial use, distribution, and reproduction in any medium, provided the original author(s) and the source are credited.

\section{REFERENCES}

1. Olysio (simeprevir) tablet package insert. Titusville: Janssen Therapeutics, Division of Janssen Products, LP; 2013.

2. Sovaldi (sofosbuvir) package insert. Foster City: Gilead Sciences; 2013.

3. Recommendations for Testing, Managing, and Treating Hepatitis C. American Association for the Study of Liver Diseases/Infectious Diseases Society of America, with International Antiviral SocietyUSA. http://www.hcvguidelines.org/full-report-view. Accessed Sept 1, 2014.

4. Greene M, Steinman MA, McNicholl IR, Valcour V. Polypharmacy, drug-drug interactions, and potentially inappropriate medications in older adults with human immunodeficiency virus infection. J Am Geriatr Soc. 2014;62(3):447-53. 
5. Centers for Disease Control \& Prevention. Division of Viral Hepatitis. Surveillance for Viral HepatitisUnited States, 2011. http://www.cdc.gov/hepatitis/ Statistics/2011Surveillance/index.htm. Accessed Sept 1, 2014.

6. Orlando G, Meraviglia P, Cordier L, Meroni L, Landonio S, Giorgi R, et al. Antiretroviral treatment and age-related comorbidities in a cohort of older HIV-infected patients. HIV Med. 2006;7(8):549-57.

7. Marks M, Kulasegaram R. Drug-drug interactions and drug resistance will limit access to treatment in patients with HIV and hepatitis $C$ virus coinfection. Clin Infect Dis. 2013;57(1):156-7.

8. Sulkowski MS. Hepatitis C virus-human immunodeficiency virus coinfection. Liver Int. 2012;32(Suppl 1):129-34.

9. Patel N, Veve M, Bliss S, Nasiri M, McNutt LA, Lazariu V, et al. Prevalence and predictors of important telaprevir drug interactions among patients coinfected with hepatitis $\mathrm{C}$ and human immunodeficiency virus. J Pharm Technol. 2014;30(5):159-67.

10. Patel N, Veve M, Nasiri M, Bliss S, McNutt LA, Lazariu V, et al. Predicting the probability of experiencing clinically significant drug-drug interactions involving boceprevir-containing hepatitis C therapy among patients coinfected with hepatitis $\mathrm{C}$ and HIV. AIDS Patient Care STDS. 2014;28(10):513-6.

11. Nachega JB, Hsu AJ, Uthman OA, Spinewine A, Pham PA. Antiretroviral therapy adherence and drug-drug interactions in the aging HIV population. AIDS. 2012;31(26 Suppl 1):S39-53.

12. Up-to-Date, Inc. Lexi-interact online. Available from http://www.uptodate.com/crlsql/interact/ frameset.jsp. Accessed Feb 1, 2014.

13. Brennan T, Shrank W. New expensive treatments for hepatitis C infection. JAMA. 2014;312(6):593-4.

14. Seminerio MJ, Ratain MJ. Preventing adverse drugdrug interactions: a need for improved data and logistics. Mayo Clin Proc. 2013;88(2):126-8.

15. Patel N, Abdelsayed S, Veve M, Miller CD. Predictors of clinically significant drug-drug interactions among patients treated with nonnucleoside reverse transcriptase inhibitor-, protease inhibitor-, and raltegravir-based antiretroviral regimens. Ann Pharmacother. 2011;45(3):317-24.

16. Parikh ND, Levitsky J. Hepatotoxicity and drug interactions in liver transplant candidates and recipients. Clin Liver Dis. 2013;17(4):737-47 (x-xi).

17. Glotzbecker B, Duncan C, Alyea E 3rd, Campbell B, Soiffer R. Important drug interactions in hematopoietic stem cell transplantation: what every physician should know. Biol Blood Marrow Transpl. 2012;18(7):989-1006.

18. Pai AB, Cardone KE, Manley HJ, St Peter WL, Shaffer $\mathrm{R}$, Somers $\mathrm{M}$, et al. Medication reconciliation and therapy management in dialysis-dependent patients: need for a systematic approach. Clin J Am Soc Nephrol. 2013;8(11):1988-99. 